\title{
UCHUVA (Physalis peruviana L.) ECOTIPO COLOMBIA, MÍNIMAMENTE PROCESADA INOCULADA CON LA CEPA \\ NATIVA Lactobacillus plantarum LPBM10 MEDIANTE LA TÉCNICA DE IMPREGNACIÓN A VACÍO
}

\section{CAPEGOOSEBERRY (Physalis Peruvian L.) COLOMBIAN ECOTYPE, MINIMALLY PROCESSED INOCULATED WITH NATIVE STRAIN Lactobacillus plantarum LPBM10 BY MEANS OF VACCUM IMPREGNATION TECHNIQUE}

\author{
Zaira Tatiana Marín A. (1), Misael Cortés R. (1), Olga Inés Montoya C. (2)
}

(1) Departamento de Ingeniería Agrícola y de Alimentos, Facultad de Ciencias Agropecuarias,

Universidad Nacional de Colombia, Sede Medellín, Colombia

(2) Escuela de Biociencias, Facultad de Ciencias,

Universidad Nacional de Colombia, Sede Medellín, Colombia.

\begin{abstract}
During the last decade the development of functional food has grown as the demand has increased significantly. The Group Functional Foods Research (GAF) of the National University of Colombia, Headquarters Medellin; applies the matrix engineering as the methodology to obtain these, adding antioxidant vitamins, minerals, probiotics, among others, in the structure of the fruits. Aim of this study was to develop capegooseberry minimally processed inoculated with Lactobacillus plantarum in glucose solution, using it as mechanism of incorporating the vacuum impregnation technique. Impregnated samples and stored at $4^{\circ} \mathrm{C}$, at time $0,5,10$ and 15 days, counting showed 1,52 $\pm 0,6 \times 10^{9} \mathrm{CFU} / 100 \mathrm{~g}$ fresh capegooseberry. Having dairy products as a referent to consider food as probiotic (10 $10^{6} \mathrm{CFU} /$ day), an agreement can be made based on the results. The fruit capegooseberry inoculated with L. plantarum presents these characteristics, providing an improvement in the consumer's diet. The development of innovative products like fruits minimally processed with probiotic microorganisms represents an advance in research and development that reinforces the growing culture food consumption that favors health.
\end{abstract}

Key words: Probiotics; capegooseberry; functional foods; vacuum impregnation; Lactobacillus plantarum.

Este trabajo fue recibido el 10 de Marzo de 2009, aceptado con modificaciones el 10 de Marzo de 2010 y aceptado para ser publicado el 9 de Noviembre de 2010.

\section{INTRODUCCIÓN}

La sociedad actual, está interesada en la búsqueda de alimentos que proporcionen un beneficio adicional para la salud, dentro de estos encontramos los alimentos funcionales (AF), que son alimentos naturales, transformados o ingredientes alimentarios a los cuales se les ha incorporado, aumentado, substituido o eliminado algún (os) componente (es) con actividad fisiológica (CFA), y contribuyen en la prevención de ciertas enfermedades $(1,2)$.
El desarrollo de AF con microorganismos probióticos ha sido enfocado principalmente en los productos lácteos, que contienen microorganismos vivos que al ser ingeridos en cantidades suficientes, ejercen un efecto positivo en la salud más allá de los efectos nutricionales básicos (3-5). El uso de microorganismos probióticos estimulan el crecimiento y desarrollo de la microbiota natural, provocando la salida de las bacterias potencialmente dañinas, la disminución del nivel de colesterol en la sangre, refuerzan los mecanismos de defensa naturales 
del cuerpo, evitan la formación de tumores (3-18), producen nutrientes y vitaminas, mejoran la biodisponibilidad de minerales y oligoelementos, estimulan la motilidad intestinal, evitan el estreñimiento y sintetizan enzimas digestivas como la $\beta$-galactosidasa que ayudan a desdoblar la lactosa $(9,13,14)$. Todos estos efectos mejoran la salud del huésped, considerando que para obtener estos beneficios su consumo debe ser superior a 106 Unidades Formadoras de Colonias/día (UFC/día) (3, 6, 7, 11, 16, $17,19,20)$. Para que el probiótico se pueda implantar o colonizar se requiere de un sustrato disponible rico en fibra u otros componentes.

Colombia es el primer productor y exportador en el mundo de uchuva (Physalis peruviana L.) seguido de Sudáfrica. El fruto se caracteriza por tener una mejor coloración y mayor contenido de azúcares (21). La uchuva es un fruto exótico con propiedades farmacológicas atribuidas principalmente a la presencia de múltiples withanólidos (lactonas esteroidales) compuestos químicos reconocidos por sus propiedades citotóxicas contra diferentes tipos de cáncer entre ellos el de seno (22-26) Los beneficios atribuidos en la medicina tradicional a la uchuva, se atribuyen al contenido de vitaminas, proteínas, minerales (21) y ácidos (principalmente oleico y linoléico) $(27,28)$; se resalta la purificación de la sangre, fortificación del nervio óptico, control de la amibiasis, calcificación de los huesos, antimicobacterial, antipirético, entre otros $(29,30)$.

Estudios realizados por algunos investigadores reportan propiedades antioxidantes $(30,31)$, antitumorales, hepatoprotectoras (32), inmunomodulatorias y antibacteriales (33), presencia en extractos y fracciones del cáliz de compuestos con actividad antiinflamatoria como el Pp-D28-LF $(31,34)$ y un efecto antiproliferativo sobre células cancerígenas pulmonares (35). Otras investigaciones, indican que el consumo de la uchuva reduce los niveles de glucosa en sangre a los 90 y 120 minutos postprandial en adultos jóvenes, causando un gran efecto hipoglicémico después de ese periodo (36); por otro lado, propiedades antihepatotóxicas de la uchuva han sido reportadas en estudios en ratas (37).

La Ingeniería de Matrices es una herramienta de la Ingeniería de Alimentos que utiliza los conocimientos sobre composición, estructura y propiedades de la matriz estructural del alimento para producir y controlar cambios que mejoren sus propiedades funcionales $\mathrm{y} / \mathrm{o}$ sensoriales (38). Esta herramienta utiliza la técnica de impregnación al vacío (IV) que incorpora los CFA en el alimento, por medio del mecanismo hidrodinámico (MHD), como un proceso de transporte de materia en un sistema sólido-poroso-líquido, el cual promueve cambios en las propiedades mecánicas, estructurales, ópticas y térmicas que afectan la calidad de frutas y vegetales y además permite el desarrollo de alimentos funcionales $(3,38-52)$.

La presente investigación tiene como objetivo desarrollar un producto mínimamente procesado a partir de uchuva inoculada con la cepa nativa Lactobacillus plantarum LPBM10, a través de la técnica IV como mecanismo de incorporación.

\section{MATERIALES Y MÉTODOS}

Materia prima. Se utilizaron uchuvas (Physalis peruviana L.) ecotipo Colombia, con pesos entre 6 y 7 g, procedentes del municipio de la Unión (Antioquia, Colombia), las cuales se desinfectaron con solución de hipoclorito de sodio a $100 \mathrm{ppm}$.

Microorganismo y medio de cultivo. Se utilizó el Lactobacillus plantarum LPBM10, microorganismo aislado a partir de repollo fermentado en el laboratorio de Microbiología Industrial de la Universidad Nacional de Colombia Sede Medellín; el cual se activó en caldo Man Rogosa y Sharpe (MRS) a $37^{\circ} \mathrm{C}$, durante $72 \mathrm{~h}$, bajo condiciones microaerófilas.

Propiedades fisicoquímicas. Se realizaron pruebas de acidez por titulación con $\mathrm{NaOH} 0,1 \mathrm{~N}$, utilizando como indicador fenolftaleína (53), de $\mathrm{pH}$ con un potenciómetro Schott CG840B (54), sólidos solubles con un refractómetro Leica auto ABBE (escala 0-32 ${ }^{\circ}$ Brix) (53), contenido de humedad (53), actividad de agua (aw) con un higrómetro AquaLAB Decagon serie 3TE de punto de rocío $25^{\circ} \mathrm{C}(53)$, densidad aparente (masa de la muestra y volumen desplazado) y la densidad del liquido de impregnación por el método del picnómetro a $20^{\circ} \mathrm{C}(54)$. La valoración de las propiedades fisicoquímicas para las uchuvas frescas se realizó en tres lotes de 30 muestras por lote y las impregnadas de 30 muestras por condición de almacenamiento.

Solución de impregnación (SI). Se utilizó una solución isotónica de glucosa con igual aw que la uchuva fresca, correspondiente a una concentración del 14\% $\mathrm{p} / \mathrm{p}$. Esta solución fue inoculada con el microorganismo en concentraciones de 0,5 (150 millones bacterias $/ \mathrm{mL})$ y 5 (1500 millones bacterias/mL), según el patrón de McFarland, con absorbancias de 0,189 y 1,307 medidas por espectrofotometría a $560 \mathrm{~nm}$ respectivamente, en un Spectronic 20 Genesys.

Proceso de impregnación a vacío (IV). Las uchuvas frescas (UF) se pesaron y sumergieron en la SI, posteriormente, se llevaron a la cámara de impregnación a una presión de vacío de 20 kpa durante 5 min, luego se restableció la presión atmosférica manteniéndolas sumergidas por 5 min más. Durante el proceso IV se evaluó la evolución de la masa de las muestras y la SI, lo 
que permitió determinar los parámetros de impregnación en cada etapa: la fracción volumétrica de impregnación (m3SI/m3UF) al final de la etapa de vacío (X1) y al final del proceso $(\mathrm{X})$, deformación volumétrica al final de la etapa de vacío ( $\mathrm{g} 1)$ y al final del proceso (g) y la porosidad efectiva (ee) disponible para el proceso IV (55). La metodología y el cálculo de los parámetros de impregnación, ha sido descrito según el modelo matemático de algunos autores $(1,55)$. Los parámetros de impregnación se determinaron a partir de 25 repeticiones por cada SI.

Recuento en placa. Cada uchuva impregnada (UI) fue homogeneizada con peptona universal $(0,1 \% \mathrm{p} / \mathrm{v})$ en proporción 1:9, luego se realizó el método de siembra en profundidad en agar MRS, las cajas de petri se incubaron a $37^{\circ} \mathrm{C}$, por $72 \mathrm{~h}$ en condiciones de anaerobiosis (56). Los conteos de células viables se realizaron como UFC $100 \mathrm{~g}$ de UF y los resultados se expresaron en unidades logarítmicas (Log10 UFC/100g UF). Se realizaron 20 repeticiones para cada tiempo de almacenamiento.

Almacenamiento. Las UI se almacenaron a $4^{\circ} \mathrm{C}$ durante los tiempos de control de 0, 5, 10 y 15 días, envasadas en condiciones atmosféricas en bolsas plásticas con multicapa de poliamida-polietileno marca ALICO S. A., con barrera al vapor de agua $\left(<15 \mathrm{~g} / \mathrm{m}^{2} / 24 \mathrm{hr} / \mathrm{atm}\right.$, $\left.\mathrm{T}=38^{\circ} \mathrm{C}\right), \mathrm{O} 2\left(60 \mathrm{cc} / \mathrm{m}^{2} / 24 \mathrm{hr} / \mathrm{atm}, 23^{\circ} \mathrm{C}\right), \mathrm{N}_{2}$ y CO $\mathrm{CO}_{2}$. Se evaluó la evolución de la estabilidad de las UI en cuanto a la población del L. plantarum, los cambios fisicoquímicos, color y textura. Se realizaron 20 repeticiones para los recuentos y 30 para el resto de parámetros por condición de almacenamiento.

Microscopia. El análisis microestructural se realizó en un microscopio electrónico de barrido (SEM) marca JEOL referencia JSM 5950 LV. Las muestras de los sustratos inoculados, son ubicadas y distribuidas a lo largo de un portaobjeto, luego se secan al ambiente, se recubren con oro y se someten a las condiciones de operación del equipo ( $25 \mathrm{~Pa}$ de vacío y $15 \mathrm{kv}$ de corriente eléctrica). Se tomaron micrografías a diferentes aumentos para identificar los microorganismos en las estructuras de estudio.

Color. Se determinó utilizando el espectrocolorímetro X-RITE, modelo SP64, el iluminante D65 y el observador de $10^{\circ}$ como referencia; las medidas se realizaron con componente especular incluida. A partir de los espectros de reflexión se obtuvieron las coordenadas de color del CIE-L*a*b, donde $\mathrm{L}^{*}$ es un indicador de la luminosidad, $\mathrm{a}^{*}$ (cromaticidad verde (-) a rojo (+)) y b* (cromaticidad azul (-) a amarillo (+)). Se midieron 30 replicas por condición de almacenamiento y para cada muestra se realizaron tres lecturas en la zona ecuatorial, una en el entorno del pedúnculo y la otra en el ápice.

Textura. Se realizaron ensayos de punción en un analizador de textura TA.XT2 (Stable Micro Systems). Se utilizó un embolo metálico plano de $5 \mathrm{~mm}$ de diámetro a una velocidad de penetración de $4 \mathrm{~mm} / \mathrm{s}$, hasta una distancia de $10 \mathrm{~mm}$. La dureza se cuantificó en términos de la fuerza máxima alcanzada a la ruptura de la película del fruto $\left(\mathrm{F}_{\mathrm{max}}\right)$, además se determinó la fuerza media de la pulpa $\left(\mathrm{F}_{\text {media }}\right)$ y el modulo de extensibilidad $\left(\varepsilon^{*}\right)$, este último valorado de acuerdo a la pendiente inicial de la curva fuerza versus distancia. Se realizaron 30 mediciones por tiempo de almacenamiento.

Análisis de datos. Los resultados fueron analizados a partir del ANOVA, utilizando el método LSD (Mínimas Diferencias Significativas) como método de comparaciones múltiples, con un nivel de confianza del $95 \%$ $(\alpha=0,05)$. El análisis de varianza fue realizado con el paquete estadístico STATGRAPHICS PLUS versión 5.1.

\section{RESULTADOS Y DISCUSIÓN Caracterización fisicoquímica}

La tabla 1 presenta los resultados promedios y las desviaciones estándar de los parámetros fisicoquímicos ${ }^{\circ} \mathrm{Brix}, \mathrm{pH}$, acidez (\%), contenido de humedad (\%) y densidad aparente de la UF y la UI a los tiempos de control durante el almacenamiento. Los valores obtenidos en las UF concuerdan con los valores reportados por otras investigaciones (57-63). Estos resultados, permiten clasificar el fruto entre "Extra" y I para el CODEX Alimentarius (64) o en la escala 3 - 4 para la Norma Técnica Colombiana (65).

No se presentaron diferencias significativas (NS) debidas al factor de impregnación ( $p>0,05)$ en ninguno de los parámetros, sin embargo se observó una pequeña disminución de los ${ }^{\circ} \mathrm{Brix}$, lo cual se atribuye principalmente al contenido de agua incorporada con la solución de impregnación (aproximadamente 86\%).

En general, todos los parámetros fueron NS $(p>0,05)$ con respecto al factor tiempo (05 IV, 10 IV y 15 IV), sin embargo, se observó en los ${ }^{\circ}$ Brix, una tendencia a disminuir, debido a que los azúcares son la fuente de carbono y energía de los microorganismos $(66,67)$, al igual que por el proceso de respiración de la fruta (68). Para el caso de los ligeros cambios de $\mathrm{pH}$ y acidez, se asocia principalmente a dos situaciones, una que hace que la acidez tienda a incrementar por la generación de acido láctico procedente de los procesos microbianos (14) y la otra que el $\mathrm{pH}$ aumente por la disminución de la intensidad respiratoria (58). La resultante es un ligero incremento tanto en el pH como en la acidez.

\section{Impregnación a vacío}

El proceso IV aplicado en la UF, presenta una característica especial, debido a la configuración estructural 
propia de la fruta. La película cérea (resina terpénica) que cubre la superficie en un $95 \%$ aproximadamente, presenta poca permeabilidad al intercambio de fluidos a través de ella, esto representa un obstáculo para el proceso IV. Sin embargo, el área del pedúnculo, correspondiente al punto de ruptura del capacho, presenta una microestructura muy porosa, que ayuda al proceso de transferencia de masa durante la impregnación del fruto.
Para analizar la respuesta a la IV, se evaluó el efecto del tamaño del fruto con respecto al área del pedúnculo (figura 1), se observó que no existe una tendencia o correlación definida entre estas variables, lo que hace más complejo el análisis de la respuesta de impregnación; esta situación permitió identificar que el área de la película cérea influye en la incorporación de líquido de impregnación en la estructura.

\section{TABLA 1}

Caracterización fisicoquímica de uchuva fresca e impregnada con L. plantarum.

\begin{tabular}{lccccc} 
Parámetro & UF & UI 0-IV & UI 05-IV & UI 10-IV & UI 15-IV \\
\hline 'Brix & $14,3 \pm 0,8$ & $13,1 \pm 0,7$ & $12,8 \pm 0,6$ & $13,0 \pm 0,7$ & $13,4 \pm 0,6$ \\
$\mathrm{pH}$ & $3,39 \pm 0,06$ & $3,36 \pm 0,08$ & $3,42 \pm 0,04$ & $3,46 \pm 0,1$ & $3,51 \pm 0,09$ \\
Acidez (\%) & $2,05 \pm 0,15$ & $2,00 \pm 0,26$ & $2,05 \pm 0,19$ & $2,07 \pm 0,14$ & $2,33 \pm 0,24$ \\
$\mathrm{a}_{\mathrm{w}}$ & $0,988 \pm 0,002$ & ----- & ----- & ----- & ----- \\
Humedad (\%) & $79,5 \pm 2,8$ & ----- & ---- & ---- & ---- \\
Densidad aparente (g/cc) & $1,038 \pm 0,054$ & ----- & ---- & --- & - \\
Peso & $6,56 \pm 0,53 \mathrm{~g}$. & & & &
\end{tabular}

UF: Uchuva fresca

0- IV: Uchuva recién impregnada

05- IV: Uchuva impregnada con 5 días de almacenamiento.

10 -IV: Uchuva impregnada con 10 días de almacenamiento.

15 -IV: Uchuva impregnada con 15 días de de almacenamiento.

\section{FIGURA 1}

\section{Relación entre el área del pedúnculo y el peso de la UF.}

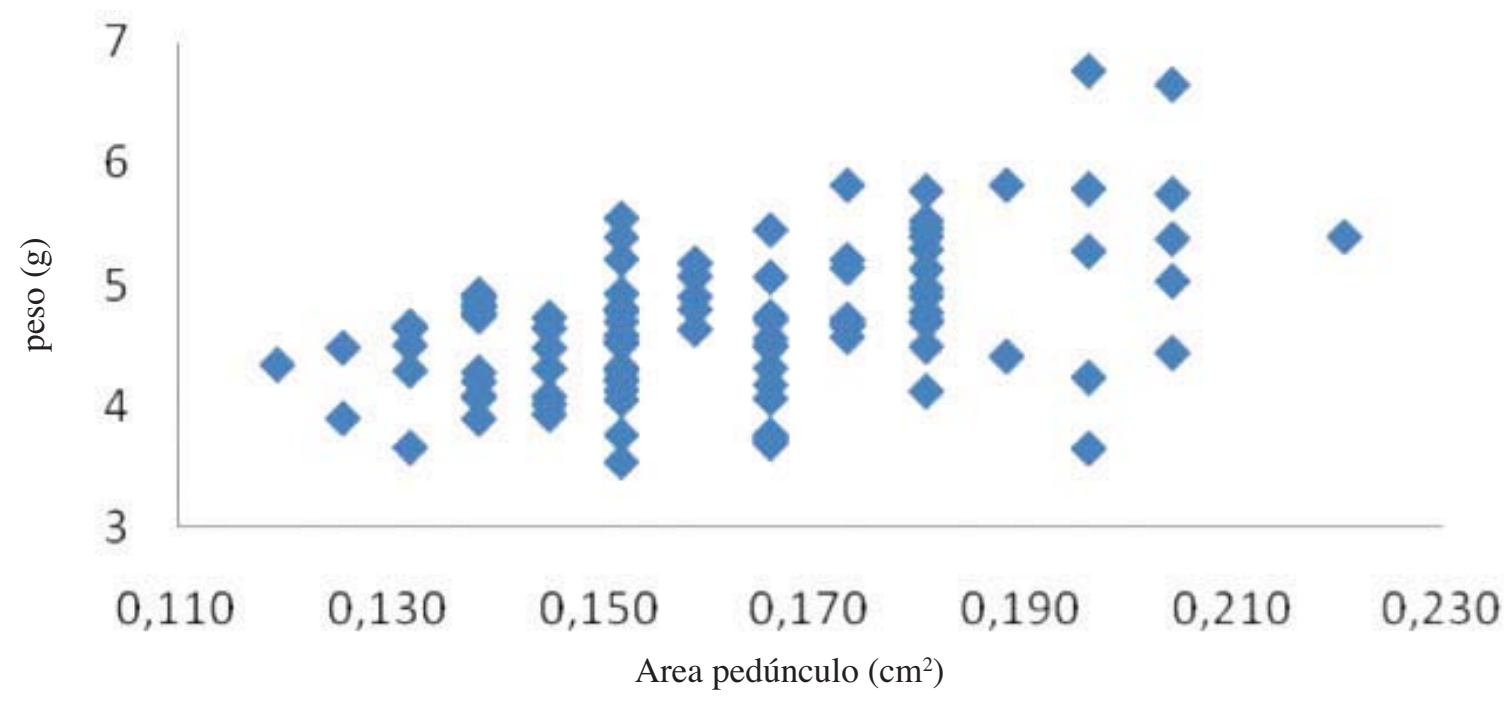


La tabla 2 presenta los valores promedios y sus desviaciones estándar de los parámetros de impregnación $\mathrm{X}, \mathrm{X}_{1}, \gamma, \gamma_{1} \mathrm{y} \varepsilon_{\mathrm{e}}$, en muestras recién impregnadas con las SI a una concentración patrón de McFarland 0,5 y 5.

En ambas concentraciones se presentan valores negativos de $\mathrm{X}_{1}$ y $\gamma_{1}$, lo que nos indica salida de líquido nativo de la estructura del fruto y una deformación volumétrica de la muestra en la etapa de vacío respectivamente.

El ANOVA fue NS $(p>0,05)$ para X1, g1 y $\varepsilon e(p=$
$0,1875, \mathrm{p}=0,7492$ y $\mathrm{p}=0,0876$ respectivamente) debido a los coeficientes de variabilidad observados; en cambio si hay diferencias significativas para $X$ y $\gamma(p=0,0000$, $\mathrm{p}=0,0474$ respectivamente). $X$ tiene valores superiores en la SI a una concentración patrón McFarland 0,5 que en la de 5, esto se asocia a la menor población de microorganismos presentes en la solución, que hace que esta sea menos viscosa y disminuya la caída de presión en los espacios intercelulares del área del pedúnculo. Por otro lado $\gamma$ está muy afectada por el MHD, la cual

\section{TABLA 2}

Respuesta a la impregnación al vacío de la Uchuva inoculada con L. plantarum.

\begin{tabular}{lcccccc} 
L. plantarum & $\begin{array}{c}\text { Peso } \\
\mathbf{U F}\end{array}$ & $\begin{array}{c}\mathbf{X} \\
\left(\mathbf{m}^{3} \mathbf{S I} / \mathbf{m}^{3} \mathbf{U F}\right)\end{array}$ & $\begin{array}{c}\mathbf{X}_{1} \\
\left(\mathbf{m}^{3} \mathbf{S I} / \mathbf{m}^{3} \mathbf{U F}\right)\end{array}$ & $\begin{array}{c}\gamma_{1} \\
\left(\mathbf{m}^{3} / \mathbf{m}^{3} \mathbf{U F}\right)\end{array}$ & $\begin{array}{c}\gamma \\
\left(\mathbf{m}^{3} / \mathbf{m}^{3} \mathbf{U F}\right)\end{array}$ & $\begin{array}{c}\boldsymbol{\varepsilon}_{\mathrm{e}} \\
\left(\mathbf{m}^{3} \mathbf{a i r e}^{\mathbf{3}} \mathbf{\mathbf { m } ^ { 3 }} \mathbf{U F}\right)\end{array}$ \\
\hline$[0,5]$ & $6,26 \pm 0,23$ & $9,63 \pm 3,58$ & $-4,72 \pm 3,35$ & $-12,03 \pm 3,43$ & $-1,59 \pm 4,79$ & $11,18 \pm 4,41$ \\
{$[5]$} & $6,96 \pm 0,44$ & $5,35 \pm 1,92$ & $-3,12 \pm 4,47$ & $-12,30 \pm 2,00$ & $-3,97 \pm 2,65$ & $9,14 \pm 3,21$
\end{tabular}

[0,5]: Concentración McFarland de 0,5

[5]: Concentración McFarland de 5

X: Fracción volumétrica de impregnación al final del proceso

$\mathrm{X}_{1}$ : Fracción volumétrica de impregnación al final de la etapa de vacio

$\gamma_{1}$ : Deformación volumétrica al final de la etapa de vacio

$\gamma$ : Deformación volumétrica al final del proceso

$\varepsilon_{\mathrm{e}}$ : Porosidad efectiva disponible para el proceso de impregnación

FIGURA 2

Valores medios con intervalos LSD (95\%) de la población de Lactobacillus plantarum LPBM10 Log UFC/100 g UF durante el almacenamiento a $4^{\circ} \mathrm{C}$.

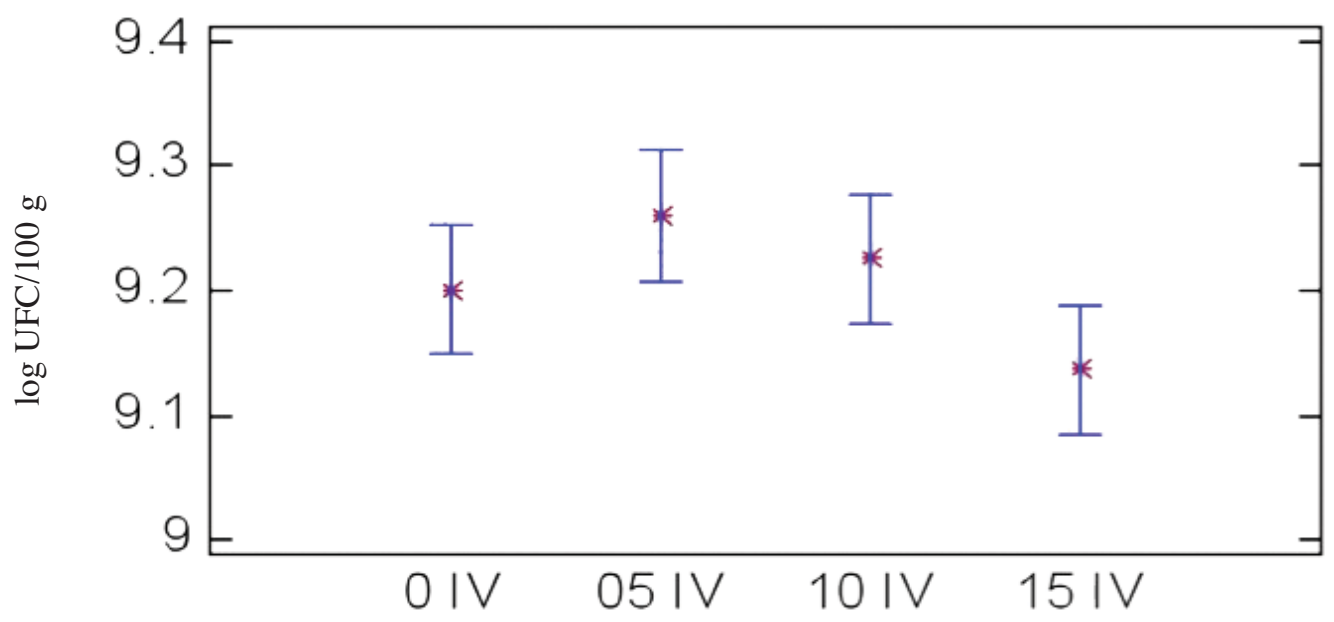

Tiempo (días) 
se favorece por la misma plasticidad de la película externa. Los valores encontrados de $\varepsilon_{\mathrm{e}}(\cong 10 \%)$, denotan la disponibilidad de este fruto para la incorporación en su interior de los CFA.

No existen investigaciones realizadas en procesos de IV en uchuva, donde se determinen los parámetros en cada etapa, sin embargo; impregnaciones con soluciones de isotónicas de sacarosa $20^{\circ}$ Brix $(X=3,2 \pm$ $\left.1,4 \% ; \varepsilon_{\mathrm{e}}=4,77 \pm 2,14 \%\right)(57,69)$, con emulsiones de vitamina $\mathrm{E}\left(\mathrm{X}=2,40 \%, \varepsilon_{\mathrm{e}}=3,20 \%\right)(57)$; con emulsiones de vitaminas $C$, E y Calcio ( $X=2,67 \pm 1,06 \%, \varepsilon_{\mathrm{e}}=4,07$ $\pm 1,6 \%$ ) (69). Estos valores inferiores a los alcanzados, se atribuyen principalmente a que no fueron considerados cambios de masa en la etapa de vacío $\left(X_{1}=0\right)$ y se consideraron nulas las deformación en ambas etapas $\left(\gamma_{1}=0, \gamma=0\right)$.
Almacenamiento

La figura 2 muestra los valores medios con intervalos LSD (95\%) de la población de microorganismos durante el almacenamiento, el ANOVA reflejó diferencias estadísticas significativas con respecto al tiempo $(\mathrm{p}=0,1263<0,05)$, sin embargo se observó que durante los primeros 10 días, los resultados se comportaron como un grupo homogéneo, con niveles entre 9,15 y 9,3 ciclos $\log _{10} / 100$ g UF y a los 15 días de almacenamiento se observó que el L. plantarum tiene tendencia a disminuir, pero conservando los niveles para ser considerado un alimento enriquecido con probióticos $(9,14 \pm 0,23$ ciclos $\log$ UFC/100g UF o $1,52 \pm 0,6$ x $10^{9}$ UFC/100 g UF).

En la actualidad existen pocas investigaciones de frutas inoculadas con probióticos, algunos autores han ensayado la incorporación de L. casei y Saccharomyces

\section{FIGURA 3}

Evolución los parámetros de color $\mathrm{L}^{*}, \mathrm{a}^{*}, \mathrm{~b}^{*}$ en las zonas del pedúnculo, ecuatorial y ápice de la UF y UI con $L$. plantarum LPBM10 durante el almacenamiento a $4^{\circ} \mathrm{C}$.
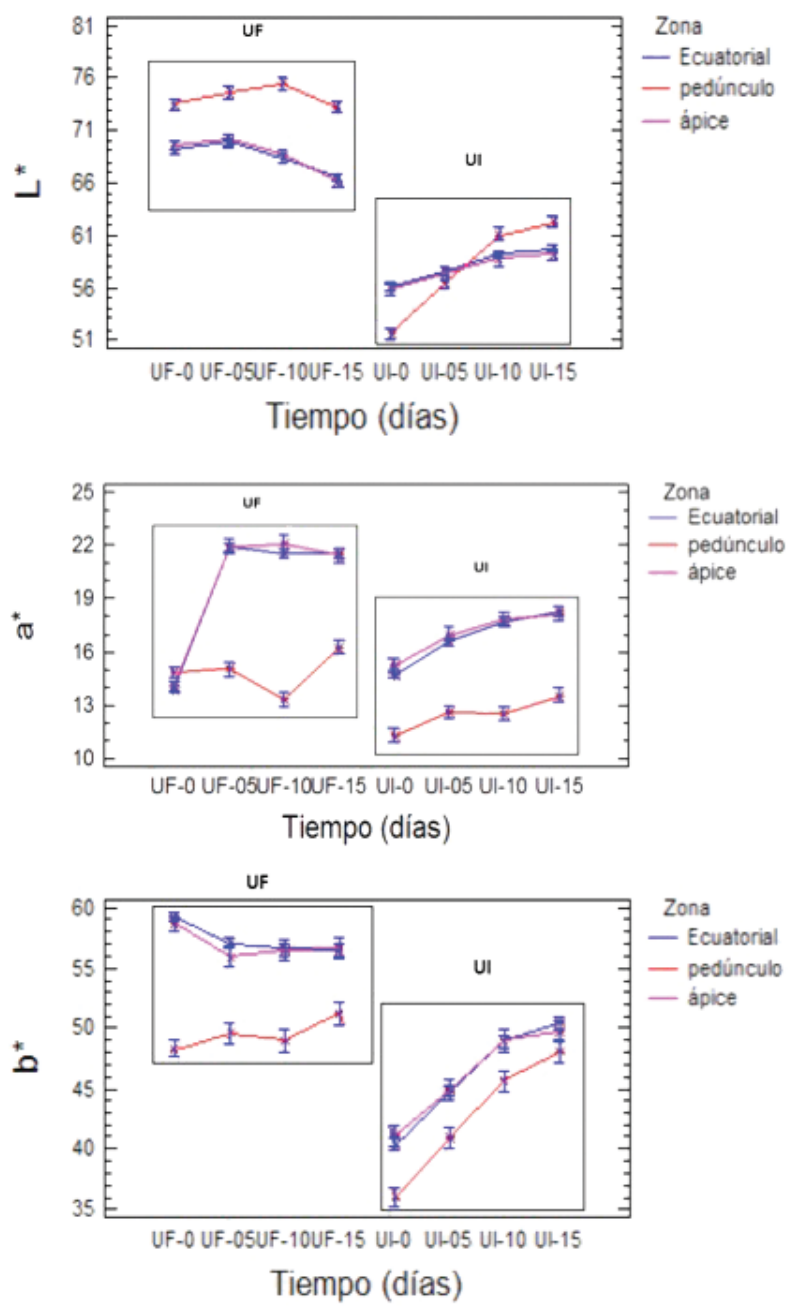
cerevisiae en manzana, utilizando la misma metodología pero alcanzando niveles máximos del orden de $1,7 \times 10^{7}$ y $2,8 \times 10^{9} \mathrm{UFC} / \mathrm{g}$ de fruta fresca respectivamente $(3$, 70). Otros autores, han impregnado mango con L.casei, alcanzando después de secar a $35^{\circ} \mathrm{C}$ entre 5 y $7 \mathrm{Log}$ UFC/g (71). En el caso de la uchuva, impregnaciones realizadas con L. acidophilus alcanzaron niveles entre 4,1 y 4,7 Log UFC/g UF (72).

La figura 3 presenta los valores medios con intervalos LSD (95 \%) de los parámetros de color en términos de $L^{*}, a^{*}, b^{*}$ durante el almacenamiento $(0,5,10,15$ días) para las UF y UI; en todos los casos se observaron, diferencias significativas $(\mathrm{p}<0,05)$ por efecto del proceso IV (cuadros separados), por el tiempo y la posición del fruto, esto asociado a la presencia de la SI en los espacios intercelulares del fruto.

Para las UF, se observaron 2 grupos homogéneos, uno conformado por las muestras evaluadas en el entorno al pedúnculo y el otro, por las zonas ecuatoriales y el ápice. En todos los tiempos, las muestras de la zona del pedúnculo son más claras $\left(>\mathrm{L}^{*}\right)$, menos rojizas $\left(<\mathrm{a}^{*}\right)$ y amarillas $\left(<b^{*}\right)$ que las otras zonas; este comportamiento se atribuye, por un lado a la menor concentración de carotenos en el área del pedúnculo y a los cambios fisiológicos durante el proceso de maduración del fruto, el cual se estabiliza a los 5 días, donde se acentúa el color anaranjado.

En general, la presencia del líquido de impregnación en los espacios intercelulares de las UI hace más homogéneo el índice de refracción, potenciando la absorción de la luz en la zona superficial, lo que hace que las muestras se observan más oscuras $\left(<\mathrm{L}^{*}\right)$ que las UF y principalmente en el área del pedúnculo. En forma similar que las UF, las UI presentan los mismos 2 grupos homogéneos, siendo la zona del pedúnculo más oscuras $\left(<\mathrm{L}^{*}\right)$ y más anaranjadas $\left(>\mathrm{a}^{*} \mathrm{y}>\mathrm{b}^{*}\right)$ con el tiempo. Comportamientos similares en la luminosidad $\left(<\mathrm{L}^{*}\right)$ han sido observados en uchuva impregnadas con emulsiones de vitamina $E(57,69)$ y en otras frutas $(48,73,74)$.

La figura 4 presenta los valores medios con interva-

\section{FIGURA 4}

Evolución de $\mathrm{F}_{\text {max }}, \mathrm{F}_{\text {media }}$ y $\varepsilon^{*}$ en las UF y UI con L. plantarum LPBM10 durante el almacenamiento a $4^{\circ} \mathrm{C}$.
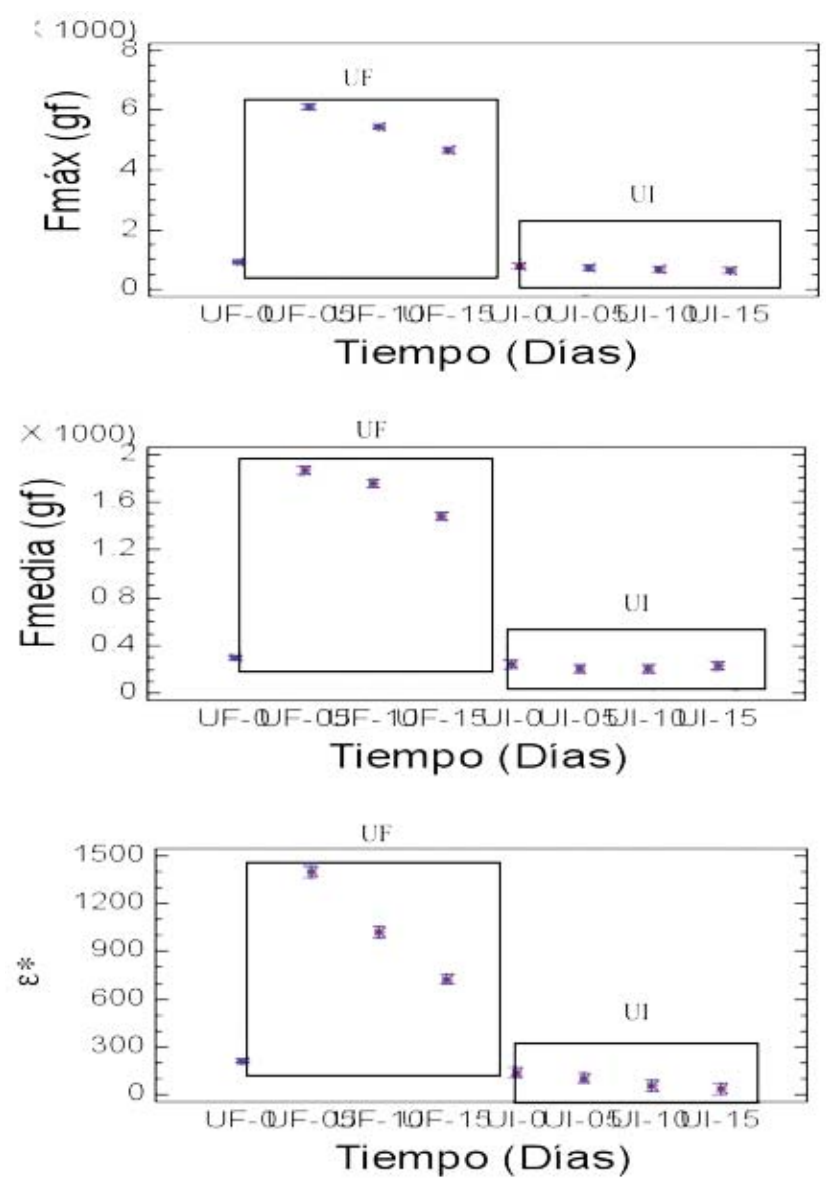
los LSD (95 \%) de la $\mathrm{F}_{\text {max }}, \mathrm{F}_{\text {media }}$ y $\varepsilon^{*}$ para las UF y UI durante el almacenamiento a $4^{\circ} \mathrm{C}$. Se observó en las UF un comportamiento similar en los parámetros de textura; igualmente ocurrió en las UI. En las UF, el ANOVA presentó diferencias significativas en todos los parámetros por efecto del factor tiempo, con un comportamiento creciente durante los primeros 5 días de almacenamiento, donde se considera que el fruto adquirió la madurez óptima. Posteriormente se presentó una disminución de los parámetros, lo cual se asoció a una sobre maduración del fruto. En las UI, el ANOVA fue NS en los parámetros Fmax, Fmedia y fue significativo para $\varepsilon^{*}$ con respecto al factor tiempo; sin embargo esta última situación se presentó en un rango de variación muy pequeño (40 - 150 $\mathrm{gf} / \mathrm{mm}$ ) y con tendencia a decrecer, es decir, las muestras se hicieron más elásticas debido a la pérdida de cohesión molecular de la película y al stress mecánico al cual se someten durante el mecanismo hidrodinámico.

\section{Microscopía}

La figura 5 presenta micrografías por SEM de la UF (a y b), UI con L. plantarum LPBM10 en el pedúnculo (c) y en la pulpa de uchuva cerca al pedúnculo (d). Las figuras a y b tomadas a 90 y 100 aumentos respectiva- mente, identificaron una microestructura totalmente distinta de la película cérea (compacta) y el pedúnculo (porosa), lo cual confirmó la hipótesis planteada inicialmente sobre la incorporación de la SI principalmente por el pedúnculo. La figura c y d tomadas a 2000 aumentos, muestra los microorganismos en el interior de la matriz, donde después de impregnado se presenta una diferencia de presión en el interior del fruto, la cual al buscar un estado de equilibrio o relajación hace que la SI se difunda al interior de la pulpa observándose grandes colonias.

\section{CONCLUSIONES}

Se evidenció una efectividad de la aplicación de la ingeniería de matrices como metodología de obtención de AF, logrando la incorporación de la cepa nativa Lactobacillus plantarum LPBM10 en la matriz estructural de la uchuva y principalmente por el pedúnculo de la misma. Los niveles de impregnación alcanzados tuvieron valores aproximados de $X$ entre 3,5 y $12 \%$ y ee entre 6 y $15 \%$. Se comprobó que el microorganismo es capaz de crecer y desarrollarse bajo condiciones extremas de baja presión $(20 \mathrm{Kpa} \cong 150,4 \mathrm{~mm} \mathrm{Hg})$, baja temperatura $\left(4^{\circ} \mathrm{C}\right)$ y tiempos de almacenamiento correspondientes a 15 días. Esto permite categorizar la uchuva impregnada con Lac-

\section{FIGURA 5}

Micrografías de la UF (a y b), pedúnculo de la UI con L. plantarum LPBM10 (c) y de la pulpa de uchuva (d) obtenidas por SEM.

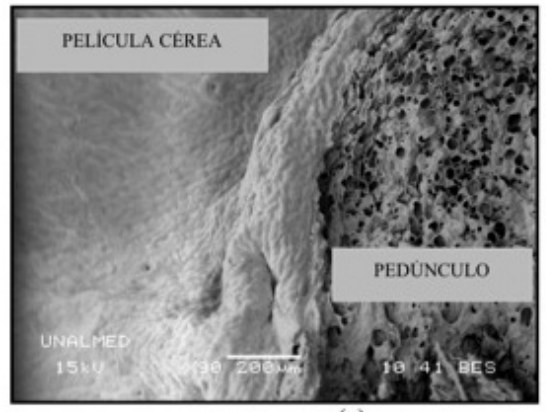

(a)

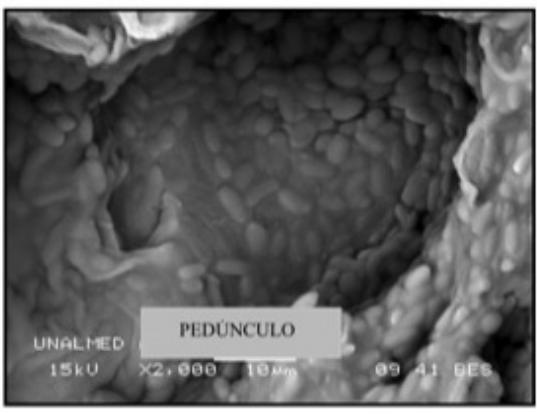

(c)

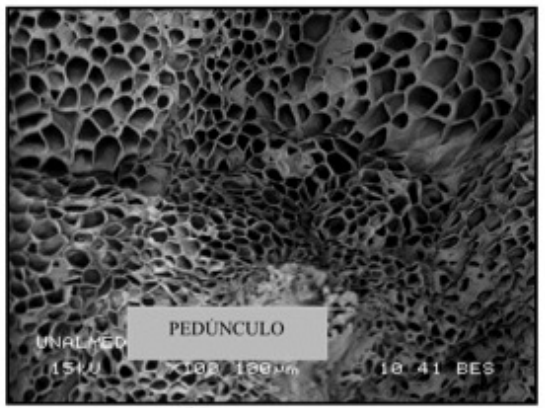

(b)

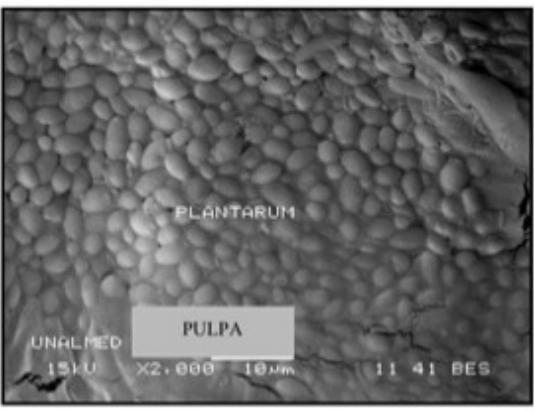

(d) 
tobacillus plantarum LPBM10 como un alimento con probióticos (promedio global: 9,21 $\pm 0,17 \log$ Ciclos de $\mathrm{UFC} / 100 \mathrm{~g}$ UF). El desarrollo de productos innovadores como frutas mínimamente procesadas enriquecidas con probióticos, representa un avance en investigación que refuerza la creciente cultura de consumo de alimentos que producen efectos benéficos en la salud.

\section{RESUMEN}

En la última década el desarrollo de alimentos funcionales ha evolucionado y su demanda se ha incrementado notablemente. El grupo de Investigación en Alimentos Funcionales (GAF) de la Universidad Nacional de Colombia Sede Medellín, aplica la Ingeniería de Matrices como metodología de obtención de estos, adicionando vitaminas antioxidantes, minerales, probióticos, entre otros, en la estructura de los frutos. El objetivo de este estudio es desarrollar uchuva mínimamente procesada, inoculada con Lactobacillus plantarum en solución de glucosa, utilizando como mecanismo de incorporación la técnica de impregnación a vacío. Las muestras impregnadas y almacenadas a $4^{\circ} \mathrm{C}$, en los tiempos de $0,5,10$ y 15 días; presentaron conteos de $1,52 \pm 0,6 \times 10^{9} \mathrm{UFC} / 100 \mathrm{~g}$ uchuva fresca. Teniendo como referente los productos lácteos para considerar un alimento como probiótico ( $10^{6} \mathrm{UFC/día),} \mathrm{se} \mathrm{puede} \mathrm{decir}$ de acuerdo con los resultados, que la uchuva inoculada con L. plantarum presenta estas características y puede proporcionar un mejoramiento en la salud del consumidor. El desarrollo de productos innovadores como frutas mínimamente procesadas con microorganismos probióticos, representa un avance en investigación que refuerza la creciente cultura de consumo de alimentos que favorecen la salud.

Palabras clave: probióticos; uchuva; alimentos funcionales; impregnación a vacío; Lactobacillus plantarum

Dirigir la correspondencia:

Profesor

Misael Cortés R.

Departamento de Ingeniería Agrícola y de Alimentos

Facultad de Ciencias Agropecuarias

Universidad Nacional de Colombia

Sede Medellín, Colombia

Fono: 57 (4) 4309070

Fax: 57 (4) 4309016.

E-mail: mcortesro@unal.edu.co

\section{BIBLIOGAFRÍA}

1. Cortés M. Desarrollo de productos de manzana deshidratados enriquecidos con vitamina E. [Tesis doctoral]. Valencia: Universidad Politécnica de
Valencia 2004.

2. Robertfroid M. Defining Functional Food. En: Gibson G, Williams CM, editors. Functional Foods: Concepts to product. Cambridge: Woodhead 2000, p. 9-29.

3. Betoret N, Puentes L, Diaz M.J, Pagán M.J, Garcia M.J, Grass M.L, Martínez-monzó J and Fito P. Development of probiotic-enriched dried fruits by vacuum impregnation. Journal of Food Engineering 2003; 56 (2-3): 273-277.

4. Tuhoy M, Probert H, Smejkal C and Gibson G. Using probiotics and prebiotic to improve gut health. Drug discovery today 2003; 8(15): 692-700.

5. Charalampopoulos D, Wang R, Pandiella $S$ and Webb C. Application of cereals and cereal components in functional foods: a review. Internat J Food Microbiol 2002; 79 (1-2): 131-41.

6. Prado F, Parada J, Pandey A and Saccol C. Trends in non-dairy probiotic beverages. Food Res Internat 2008; 41(2): 111-23.

7. Collado M, Meriluoto J and Salminen S. Measurement of agregation properties between probiotics and pathogens: in vitro evaluation of different methods. J of Microbiol Methods 2007; 71(1): 71- 4.

8. Shah N. Review Functional cultures and health benefits. Internat Dairy J 2007; 17(11): 1262- 1277.

9. Jenkins B, Holsten S, Bengmark S and Martindale R. Probiotics: A Practical Review of Their Role in Specific Clinical Scenarios. Nutr Clin Practice 2005; 20(2):262-70.

10. Pennacchia C, Ercolini D, Blaiotta G, Pepe O, Mauriello $G$ and Villani F. Selection of Lactobacillus strains from fermented sausages for their potential use as probiotics. Meat Science 2004, 67 (2):309-17.

11. Fasoli S, Marzotto M, Rizzotti L, Rossi F, Dellaglio F and Torriani S. Bacterial composition of commercial probiotic products as evaluated by PCR-DGGE analysis. Internat Food Microbiol. 2003; 82 (1): 59-70.

12. Bomba A, Nemcova R, Mudronova D and Guba P. The possibilities of potentiating the efficacy of probiotics. Trends Food Sci \& Technol 2002; 13(4): 121-26.

13. Hammes W and Hertel C. Research approaches for pre- and probiotics: challenges and outlook. Food Res Internat 2002; 35(2-3): 165-170.

14. Holzapfel W and Schillinger U. Introduction to preand probiotics. Food Res Internat. 2002; 35(2-3): 109-16.

15. Puupponen-Pimiä R, Aura A, Oksman-Caldentey K, Myllärinen P, Saarela M, Mattila-Sandholm T and Poutanen K. Development of functional ingre- 
dients for gut health. Food Sci \& Technol 2002; 13 (1): 3-11.

16. Reuter G, Klein $\mathrm{G}$ and Goldberg M. Identification of probiotic cultures in food samples. Food Res Internat 2002; 35 (2-3): 117-124.

17. Zubillaga M, Weill R, Postaire E, Goldman C, Caro $\mathrm{R}$ and Boccio J. Effect of probiotics and functional foods and their use indifferent diseases. Nutr Res. 2001; 21(3): 569- 79.

18. Salminen S and Von A. Microbiology and Functional aspects. Lactic Acid Bacteria. 2 ed. Marcel Dekker INC. 1998, p. 56- 72.

19. Lourens-Hattingh A and Viljoen B. Growth and survival of a probiotic yeast in dairy products. Food Res Internat. 2001a; 34(9): 791-6.

20. Lourens-Hatting A and Viljeon B. Yogurt as probiotic carrier food. Internat Dairy J. 2001b; 11(1-2): $1-17$.

21. Fisher G, Flórez V y Sora A. Producción, poscosecha y exportación de la uchuva. Universidad Nacional de Colombia, Facultad de Agronomía, 2000.

22. Lan Y. H Chang F.R, Pan M.J, Wu C.C, Wu S.J, Chen S.L, Wang S.S, Wu M, Wu Y.C. New cytotoxic withanolides from Physalis peruviana. Food Chem 2009; 116 (2): 462-9.

23. Piñeros Y, Otálvaro A, Velásquez M. Efecto de la aplicación de elicitores sobre la producción de 4b-hidroxiwithanólido E, en raíces transformadas de Physalis peruviana L. Universitas Scientiarum 2009. En: www.javeriana.edu.co/universitas_scientiarum. Consultado: Noviembre 3 de 2010.

24. Wu S, Ng L, Chen C, Lin D, Wang S and Lin C. Antihepatoma activity of Physalis angulata and Physalis peruviana extracts and their effects on apoptosis in human Hep G2 cells. Life Sci. 2004; 74: 2061-73.

25. Wu S, Ng L, Lin D, Huang S, Wang S and Lin C. Physalis peruviana extract induces apoptosis in human Hep G2 cells through CD95/CD95L system and the mitochondrial signaling transduction pathway. Cancer Lett 2004; 215 (2): 199-208.

26. Jayaprakasam B, Zhang Y, Seeram NP, Nair MG. Growth inhibition of human tumor cell lines by withanolides from Withania somnifera leaves. Life Sci 2003; 74 (1): 125-32.

27. Rodriguez E; Rockenbach I; Cataneo C; Gonzaga L; Chaves E; Fett R. Minerals and essential fatty acids of the exotic fruit Physalis peruviana L. Ciência e Tecnol Alimentos 2009; 29(3): 642-5.

28. Ramadan M; Mörsel J. Oil Goldenberry (Phisalis peruviana L.). Journal of Agricultural and Food Chem 2003; 51: 969-74.

29. Osorio D y Roldán J. Manual de la uchuva, Osorio,
Editor Grupo latino LTDA, 2004.

30. Wu S, Ng L, Huang Y, Lin D, Wang S, Huang S and Lin C. Antioxidant of Physalis peruviana. Biol \& Pharma Bull. 2005; 28 (6): 963-6.

31. Wu S, Tsai J, Chang S, Lin D, Wang S, Huangc S and $\mathrm{Ng}$ L. Supercritical carbon dioxide extract exhibits enhanced antioxidant and anti-inflammatory activities of Physalis peruviana. J Ethnopharmacol 2006; 108: 407-13.

32. Chang JC, Lin CC, Lin DL, Wang SS, Miaw CL, $\mathrm{Ng}$ LT. Antioxidative and hepatoprotective effects of Physalis peruviana extract against acetaminopheninduced liver injury in rats. Pharmaceutical Biol. 2008; 46: 724-31.

33. Veleiro A. S, Oberti J. C, Burton G. Chemistry and bioactivity of withanolides from South American Solanaceae. Studies in Natural Products Chemistry, (Part L) Bioactive Natural Products 2005; 32: 1019-52.

34. Franco L, Matiz G, Calle J, Pinzón R, Ospina L. Actividad antiinflamatoria de extractos y fracciones obtenidas de cálices de Physalis peruviana L. Biomédica 2007; 27 (1): 110-115.

35. Wu S.J, Chang S.P, Lin D.L, Wang S.S, Hou F.F, Ng L.T. Supercritical carbon dioxide extract of Physalis peruviana induced cell cycle arrest and apoptosis in human lung cancer H661 cells. Food Chem Toxicol. 2009; 47(6): 1132-38.

36. Rodríguez, S., \& Rodríguez, E. Efecto de la ingesta de Physalis peruviana (aguaymanto) sobre la glicemia postprandial en adultos jóvenes. Rev Méd Vallejiana 2007; 4(1): 43-52.

37. Arun M, Asha V.V. Preliminary studies on antihepatotoxic effect of Physalis peruviana Linn. (Solanaceae) against carbon tetrachloride induced acute liver injury in rats. J Ethnopharmacol 2007; 111 (1): 110-114.

38. Chiralt A, Fito P, Andrés A, Barat J, MartínezMonzó J, Martínez-Navarrete N. Vacuum impregnation: a tool in minimally processing of foods. En: Processing of Foods: Quality Optimization and Process Assesment. Eds: F.A.R. Oliveira y J.C. Oliveira. CRC Press, Boca Ratón 1999, 341-356.

39. Cortés M y Chiralt A. Cinética de los cambios de color en manzana deshidratada por aire fortificada con vitamina E. Vitae 2008; 15 (1): 8-16.

40. Cortés M, García A y Suárez H. Fortificación de Hongos comestibles (Pleurotus ostreatus) con calcio, selenio y vitamina C. Vitae 2007a; 14 (1): 16-24

41. Cortés M, Osório A y García E. Manzana deshidratada fortificada con vitamina $\mathrm{E}$ utilizando la Ingeniería de Matrices. Vitae 2007b; 14 (2): 17-26 
42. Cortés M, Guardiola L y Pacheco R. Aplicación de la ingeniería de matrices en la obtención de mango mínimamente procesado enriquecido con calcio. DYNA 2007c; 74 (153-2): 19-26

43. Cháfer M, Martínez-González C, Chiralt A, Fito P. Microstructure and vacuum impregnation response of citrus peels. Food Res Internat 2003, 36: 35-41.

44. Grass M, Vidal-Brotons D, Betoret N, Chiralt A and Fito P. Calcium Fortification of vegetables by vacuum impregnation Interactions with cellular matrix. J Food Engineering 2003, 56: 279-84.

45. Chiralt A, Martínez-Nuria N, Martínez-Monzó J, Talens P, Moraga G, Ayala A and Fito P. Changes in mechanicals properties throughout osmotic processes Cryoprotectant effect. J Food Engineering 2001, 49: 129 - 35.

46. Fito P, Chiralt A, Betoret N, Grass M, Cháfer M, Martinez-Monzo J, Andrés A and Vidal D. Vacuum impregnation and osmotic dehydration in matrix engineering application in functional fresh food development. J Food Engineering 2001a, 49: 175-83.

47. Fito P, Chiralt A, Barat J, Andrés A, MartinezMonzo J and Martínez-Navarrete N. Vacuum impregnation for development of new dehydrated products. J Food Engineering 2001b, 49 : 297-302.

48. Fito P and Chiralt A. Vacuum Impregnation of Plant Tissues. En: Design of Minimal processing technologies for Fruits and Vegetables. Ed. Alzamora S.M, Tapia M.S \& López-Malo A. Aspen Publisher, Inc. Maryland 2000, p. 189-204.

49. Martínez-Monzó J, Barat J, González-Martínez C, Chiralt A and Fito P. Changes in thermal properties of apple due to vacuum impregnation. J Food Engineering 2000, 43(4) : 213-18 .

50. Tapia M, López-Malo A, Consuegra R, Corte P and Welti-Chanes J. Minimally processed papaya by vacuum osmotic dehydration techniques. Food Sci Tech Internat 1999; 5:43-52.

51. Martínez-Monzó J. Cambios físico-químicos en manzanas Granny Smith asociados a la impregnación a vacío. Aplicaciones en congelación. [Tesis doctoral]. Universidad Politécnica de Valencia, España 1998.

52. Muntada V, Gerschenson L, Alzamora S and Castro M. Solute infusion effects on texture of minimally processed kiwifruit. J Food Sci. 1998, 63: 616-620.

53. Association of Official Analytical Chemists. AOAC. Official Methods of analysis. Basic calculations for chemical and biological analyses Bassey. J.S. Efiok. Arlington, VA. 1996.

54. Association of Official Analytical Chemists. AOAC. Official Methods of analysis 15th ed. Arlington,
VA. 1990

55. Salvatori D, Andrés A, Chiralt A and Fito P. The response of some properties of fruits to vacuum impregnation. J Food Engineering 1998; 21: 59-73.

56. Madigan M, Martinko J y Parker J. Brock Biología de los Microorganismos. Prentice Hall 10 a ed. Madrid, 2004.

57. Restrepo A. Nuevas perspectivas de consumo de frutas: uchuva (Physalis peruviana L.) y fresa (Fragaria vesca L.) mínimamente procesadas fortificadas con vitamina E [Tesis maestría en Ciencia y Tecnología de Alimentos]. Colombia: Universidad Nacional de Colombia; Sede Medellín; 2008. 109 p.

58. Lanchero O, Velandia G, Fischer G, Varela N y García H. Comportamiento de la uchuva (Physalis peruviana L.) en poscosecha bajo condiciones de atmósfera modificada activa. Rev Corpoica. Ciencia y Tecnol Agropecuaria 2007; 8 (1): 61-8.

59. Ávila J, Moreno P, Fischer G and Miranda D. Influencia de la madurez del fruto y del secado del cáliz en la uchuva Physalis peruviana L. almacenada a $18^{\circ} \mathrm{C}$. Acta Agronómica 2006, 55 (4): 29-37.

60. Novoa R, Bojaca M, Galvis J y Fisher G. La madurez del fruto y el secado del cáliz influyen en el comportamiento poscosecha de la uchuva almacenada a $12^{\circ} \mathrm{C}$ (Physalis peruviana L.). Agronomía colombiana 2006; 24 (1): 77-86.

61. Alvarado P, Verdugo C, Fisher G. Efecto de un tratamiento de frio $\left(1,5^{\circ} \mathrm{C}\right)$ y la humedad relativa sobre las características fisicoquímicas de frutos de uchuva (Physalis peruviana L.) durante el posterior transporte y almacenamiento. Agronomía Colombiana 2004; 22 (2): 147-59.

62. Castañeda G y Paredes R. Estudio del proceso respiratorio, principales ácidos orgánicos, azucares y algunos cambios fisicoquímicos en el desarrollo del fruto de la uchuva (Physalis peruviana L.). Inventario de investigaciones realizadas en poscosecha de productos agrícolas en la Universidad Nacional de Colombia, Sede Bogotá; 2003.

63. Herrera A. Manejo poscosecha. En: Flórez V, Fisher G, Sora A, Editores. Producción, poscosecha y exportación de uchuva (Physalis peruviana L.) Bogotá: Universidad Nacional de Colombia 2000 : 109-127.

64. CODEX Alimentarius. Frutas y Hortalizas frescas: Norma para la uchuva. Codex stan 226- 2001, 2007.

65. Instituto Colombiano de Normas Técnicas ICONTEC. Uchuva (Physalis peruviana), para el consumo fresco o destinado al procesamiento industrial. Norma Técnica Colombiana NTC 4580, Bogotá 1999.

66. Bustos G, De la Torre N, Martínez M, Casares C y Domarco Y. Evaluación de azucares hemicelulósi- 
cos de las podas de sarmiento y lías de vinificación como medio nutritivo para la producción de ácido láctico por Lactobacillus pentosus. Ciencia Tecnol Alimentaria 2004; 4(4): 283-291.

67. Serna L y Rodríguez A. Jugo de caña verde como sustrato en la producción fermentativa por lotes de ácido láctico. Rev Colombiana Biotecnol 2004; 6(2): 37-42.

68. Wills R, McGlasson B, Graham D y Joyce D. Postharvest: An introduction to the physiology and handling of fruit, vegetables and ornamentals. Adelaide 1998; $262 \mathrm{p}$.

69. Botero A. Aplicación de la ingeniería de matrices en el desarrollo de uchuva mínimamente procesada fortificada con calcio, vitamina C y E. [Tesis maestría Ciencias Farmacéuticas]. Colombia: Universidad de Antioquia; 2009

70. Puente L. Aplicación de la técnica de impregnación a vacío en la obtención de alimentos funcionales con contenido probiótico a partir de manzana. [Tesis doctoral]. Valencia: Universidad Politécnica de Valencia; 2003.
71. Giraldo G, Duque A y Padilla L. Impregnación de mango con probióticos. VIII Congreso de estudiantes de Química 2007, Universidad del valle, Cali.

72. Cueto M, Pardo J, García D, Quintero J y Posada I. Supervivencia de bacterias ácido lácticas en uchuvas impregnadas y deshidratadas con aire caliente. III Jornadas de Socialización de resultados de Investigación, septiembre 10 - 11, Universidad de la Sabana, 2008. En: http://enif.unisabana. edu.co/InvestigacionWeb/WFPonenciaPublica. aspx?PON=39. Consultado: 1 de diciembre de 2008.

73. Igual M, Castelló M, Ortola M, Andrés A. Influence of vacuum impregnation on respiration rate, mechanical and optical properties of cut persimmon. J Food Engineering 2008; 86 (3): 315-23.

74. Alzamora S, Gerschenson L. Effect of water activity depression on textural characteristics of minimally processed fruit. In: New Frontier in Food Engineering. Proceedings of the 5th Conference of Food Engineering. Barbosa-Canovas, G, Lombardo S, Narsimhan G, Okos M, eds. New York. AICHE; 1997; p.72-75. 